\title{
Changes in circulating lymphocyte subpopulations in pigs receiving therapeutic doses of ceftiofur and tulathromycin
}

\author{
Ewelina Czyżewska-Dors, Krzysztof Kwit, Zygmunt Pejsak, Małgorzata Pomorska-Mól \\ Department of Swine Diseases, \\ National Veterinary Research Institute, 24-100 Pulawy, Poland, \\ malgorzata.pomorska@piwet.pulawy.pl
}

Received: August 1, 2016

Accepted: November 7, 2016

\begin{abstract}
Introduction: The aim of the study was to evaluate the effect of administration of therapeutic doses of ceftiofur and tulathromycin on the circulating lymphocyte subpopulations in healthy pigs. Material and Methods: The study was conducted on thirty healthy 7- to 10-week-old pigs, assigned to three groups: the TUL group, injected with tulathromycin $(\mathrm{n}=10)$; the CEF group, injected with ceftiofur $(\mathrm{n}=10)$; and the $\mathrm{C}$ group, the control with no antibiotic administration $(\mathrm{n}=10)$. Blood samples were collected before, during, and after treatment with antimicrobials. Lymphocyte subpopulations circulating in the blood were determined by immunostaining and flow cytometry analyses. Results: Following administration of a therapeutic dose of tulathromycin, there were no changes in the lymphocyte subpopulations circulating in blood. In contrast, administration of ceftiofur at the recommended dose decreased the absolute number of $\mathrm{CD} 3+, \mathrm{CD} 21+, \mathrm{CD} 4+\mathrm{CD} 8-, \mathrm{CD} 4-\mathrm{CD} 8+$, and double positive CD4CD8 cells. Conclusion: Results from the study indicate that ceftiofur possesses the ability to modulate the immune system in healthy pigs by decreasing lymphocyte subpopulations circulating in blood.
\end{abstract}

Keywords: pigs, ceftiofur, tulathromycin, lymphocyte subsets, immunomodulation.

\section{Introduction}

Ceftiofur and tulathromycin are antibiotics approved to treat polymicrobial respiratory infection in pigs and cattle. Ceftiofur is a veterinary third-generation cephalosporin with a wide spectrum of effects against Gram-positive and Gram-negative bacteria, including $\beta$ lactamase-producing strains. Like other cephalosporins, ceftiofur exerts its antibacterial effect through the inhibition of cell wall biosynthesis. In pigs, a labelled dose $(3 \mathrm{mg} / \mathrm{kg})$ is suggested to fight respiratory infection caused by Actinobacillus pleuropneumoniae, Pasteurella multocida, Salmonella Choleraesuis, and Streptococcus suis $(4,9)$. Tulathromycin is a novel, semi-synthetic triamilide antibiotic, belonging to the macrolide class, and acting through the inhibition of essential protein biosynthesis by selective binding to bacterial 50S ribosomal subunits (10). In pigs, a single administration of tulathromycin at the recommended dose $(2.5 \mathrm{mg} / \mathrm{kg})$ is effective in antagonising infections caused by A. pleuropneumoniae, P. multocida, Borde- tella bronchiseptica, Mycoplasma hyopneumoniae, and Haemophilus parasuis $(13,19)$.

In addition to their antibacterial effects, tulathromycin and ceftiofur promote immunomodulatory reactions in the host immune system. With regard to tulathromycin, in vitro and in vivo studies have reported beneficial anti-inflammatory properties. In Mannheimia haemolytica-infected calves, treatment with recommended doses of tulathromycin increased neutrophil apoptosis, efferocytosis of dying cells, and reduced levels of proinflammatory leukotriene B4 (LTB4). The anti-inflammatory features of tulathromycin, evident through a reduction in the levels of proinflammatory interleukin-8 (IL-8), were also observed in lipopolysaccharide (LPS)-stimulated bovine neutrophils and macrophages $(15,16)$. Evidence of the proapoptotic effect of tulathromycin on leukocytes, enhanced efferocytosis process, and inhibition of LTB4 production has also been noted in A. pleuropneumoniaechallenged pigs (11). Beyond the pro-resolution effects, it has also been shown that tulathromycin promotes the 
post-vaccinal humoral immune response in pigs vaccinated against erysipelas (22).

Similar to tulathromycin, pig vaccination in the presence of ceftiofur may affect the post-vaccinal immune response. In studies by Pomorska-Mól et al. (21, 22), ceftiofur hydrochloride administered in a recommended dose significantly decreased the production of specific antibodies against Erysipelothrix rhusiopathiae and swine influenza virus (SIV). In pigs treated with ceftiofur and vaccinated at the same time against pseudorabies virus (PRV), a significant delay in the development of the humoral response as well as a reduction in the cellular immune response against PRV were observed (21). Detrimental effects of ceftiofur sodium on the post-vaccinal immune response were also noted in poultry vaccinated against Escherichia coli infection (14). In contrast, in mice with LPS-induced endotoxaemia, a beneficial effect of ceftiofur administration was reported. In endotoxic mice, ceftiofur treatment reduced the mortality rate through modulation of the inflammatory cytokine profile (10). A similar finding was observed in rats treated with cefaclor and stimulated by phytohaemagglutinin (PHA) (18). Treatment with this compound enhanced interferon- $\gamma$ (IFN- $\gamma$ ), interleukin-2 (IL-2), and interleukin-10 (IL-10) synthesis, and decreased production of interleukin-4 (IL4) and interleukin-6 (IL-6) in comparison to control rats.

Knowledge about the immunomodulatory properties of ceftiofur and tulathromycin within the immune system of challenged and/or vaccinated pigs is well-documented. However, information regarding the influence of the aforementioned antimicrobials on circulating lymphocyte subpopulations, either in nonchallenged or non-vaccinated pigs, is insufficient and unclear. Thus, modulation of the lymphocyte subpopulation may have a negative clinical implication for the host. Therefore, the objective of this study was to evaluate the effect of a therapeutic dosage of ceftiofur and tulathromycin on circulating T-cell subpopulations in clinically healthy pigs.

\section{Material and Methods}

Animals. In total, the study used 30 clinically healthy 7 - to 10-week-old pigs comprising both boars and sows with PIC genetics. The animals originated from a fully healthy herd. None of the pigs received any antibiotics before the study. The pigs were randomly assigned to three groups of ten animals each: the TUL group was injected with tulathromycin, the CEF group was injected with ceftiofur, and the $\mathrm{C}$ group received no antibiotic, serving as the control. During the experiment, the groups were housed in separated units under identical environmental conditions. Ad libitum diets (without antibiotics) were supplied. Before the study, the pigs were acclimatised for a seven-day period in which their clinical status was monitored and their ears were tagged with numbers.
Drug. The commercially available products Ceftiocyl (Vetoquinol, Biowet, Poland) and Draxxin (Zoetis, USA), containing $50 \mathrm{mg} / \mathrm{mL}$ of ceftiofur and $100 \mathrm{mg} / \mathrm{mL}$ of tulathromycin, respectively, were used.

Experimental design. Pigs from the CEF group received ceftiofur intramuscularly for $5 \mathrm{~d}$ consecutively at the recommended dose of $3 \mathrm{mg} / \mathrm{kg}$ b.w. per day. Pigs from the TUL group were administered with tulathromycin, given as a single injection (on day 0 ) at the recommended dose of $2.5 \mathrm{mg} / \mathrm{kg}$ b.w. Pigs from the $\mathrm{C}$ group were injected with PBS and used as a control. Blood samples were taken from the vena jugularis or vena cava cranialis and collected in vacuum tubes containing an EDTA-K3 anticoagulant (Medlab, Poland) on days 0 (before antibiotic administration), 4 (during ceftiofur administration), 8, and 12 after ceftiofur and tulathromycin treatment.

Haematological examinations. The total leukocyte (WBC) count and proportion of lymphocytes (LYM) in whole blood samples were measured in an Abacus Junior Vet 5 haematology analyser (Diatron, Hungary). LYM proportions were calculated as a percentage of the leukocyte count. To provide accurate results, the haematology analyser was regularly calibrated and the results were compared with reference method (microscopy) results (8).

Flow cytometry. Immunostaining and flow cytometry analyses of the lymphocyte subsets in whole blood were performed according to a previously described procedure (20). Stained cells were analysed on a FACS Canto II flow cytometer (Becton Dickinson, USA). The study used a dual-colour staining protocol, in which cells were double stained for CD3/CD21 and CD4/CD8 with the following antibodies: mouse IgG1 anti-pig CD3 (clone PPT), mouse IgG1 $\kappa$ anti-pig CD21 (clone BB6-11C9.6), mouse IgG2bא anti-pig CD4a (clone 74-12-4), and mouse IgG2aא anti-pig CD8a (clone 76-2-11) (SouthernBiotech, USA). All the mAbs were labelled with PE or FITC respectively. FITCconjugated, pan-leukocyte antigen mouse IgG1 anti-pig CD45 (clone 1E4) and monocyte antigen RPEconjugated mouse IgG2b anti-pig CD14 (clone MIL2) mAbs were used together for gating the lymphocytes (Antigenix America, USA). To determine the borderline between stained and unstained cells in the flow cytometry analysis, respective isotype controls were included in the assay. Lymphocyte subpopulations were detected by fluorochrome-labelled antibodies, according to the following definitions: CD3+ ( $\mathrm{T}$ cells), CD21+ (B cells), CD4+CD8- (helper T cells (Th)), CD4-CD8+ (cytolytic T cells (CLT)), and CD4+CD8+ (cytolytic and memory $\mathrm{T}$ cells). The outcomes were expressed as percentages of the gated lymphocytes and absolute number of cells. The percentages of cells with respective phenotypes were converted to absolute cell numbers per microlitre of blood, according to the counted lymphocytes. The computer application BD FACSDiva Software 6.0 (BD Biosciences, USA) was used for data acquisition and analysis. 
Statistical analysis. All data were expressed as a mean \pm standard deviation (SD). Data from all groups were subjected to a W Shapiro-Wilk test of normality and a Levene's test of equal variances. Due to nonnormally distributed data, the comparisons between groups at each time point were assessed using a KruskalWallis test, and the significance of changes in continuous data was evaluated by a non-parametric Friedman test. Differences with $\alpha<0.05$ were considered significant. Statistical calculations were performed using Statistica 8.0 software (Statsoft, USA).

\section{Results}

The number of WBC and LYM in pigs from all groups remained stable throughout the study $(\mathrm{P}>0.05)$ and did not differ significantly between groups $(\mathrm{P}>0.05)$. The number of WBC and the mean number of LYM in pigs from the control group ranged from $17.23 \times 10^{9} / \mathrm{L}$ to $24.32 \times 10^{9} / \mathrm{L}$ and from $7.41 \times 10^{9} / \mathrm{L}$ to $13.24 \times 10^{9} / \mathrm{L}$ respectively. In pigs from the CEF group, the number of WBC and the mean number of LYM ranged from $18.19 \times 10^{9} / \mathrm{L}$ to $25.41 \times 10^{9} / \mathrm{L}$ and from $8.03 \times 10^{9} / \mathrm{L}$ to $15.34 \times 10^{9} / \mathrm{L}$, respectively. A similar trend was observed in the TUL group, in which the numbers of WBC fell between $17.29 \times 10^{9} / \mathrm{L}$ and $26.07 \times 10^{9} / \mathrm{L}$ and the mean numbers of LYM were in the envelope $8.21 \times 10^{9} / \mathrm{L}$ to $14.95 \times 10^{9} / \mathrm{L}$. No significant differences were found with regard to the percentage of LYM in all experimental groups $(\mathrm{P}>0.05)$ (data not shown).

The mean percentage of $\mathrm{CD} 3+, \mathrm{CD} 21+$, CD4+CD8-, CD4-CD8+ cells, and the CD4:CD8 ratio (calculated as the percentage of CD4+ cells, divided by the percentage of $\mathrm{CD} 8+$ cells) in all the analysed groups did not change over time $(\mathrm{P}>0.05)$. A significant difference was only observed in the proportion of CD4+CD8+ cells in the CEF group on day 12, which was significantly lower than in the TUL and $\mathrm{C}$ groups $(\mathrm{P}<0.05)$ (Fig. 1).

The number of CD3+, CD21+, CD4+CD8-, CD4CD8+, CD4+CD8+ cells, and the CD4:CD8 ratio (calculated from the absolute size of the lymphocyte subsets) remained stable throughout the study in pigs administered with tulathromycin ( $>>0.05)$ (Fig. 2). At the same time, in the CEF group there was a significant decrease in the number of CD3+, CD21+, and CD4-CD8+ on day 8 compared to the TUL and $\mathrm{C}$ groups $(\mathrm{P}<0.05)$ (Fig. 2). A significant influence of ceftiofur on the number of CD4+CD8- and CD4+CD8+ cells was also observed. On the $8^{\text {th }} \mathrm{d}$ of the experiment, the number of CD4+CD8cells decreased compared to the TUL and C groups and to the values from day 0 . On the same day of the study, the number of $\mathrm{CD} 4+\mathrm{CD} 8+$ cells was also significantly lower than in the $\mathrm{C}$ group. A significant decrease in the number of double positive cells in pigs administered with CEF was also noted on day 4, compared to the TUL and C groups $(\mathrm{P}<0.05)$ (Fig. 2). Only the CD4:CD8 ratio remained unchanged in the CEF group during the study $(\mathrm{P}>0.05)$.

\section{Discussion}

This study was undertaken to evaluate the effects of tulathromycin and ceftiofur, the two antimicrobials frequently used in pigs, on the T-cell subpopulations circulating in blood. As far as the authors are aware, this is the first study on the influence of tulathromycin and ceftiofur provided in the recommended dosage on distinct lymphocyte subsets circulating in blood.

The outcome of the study revealed that tulathromycin administered in a therapeutic dose was neutral to WBC counts and to the counts and percentage of the T-cell subpopulations circulating in blood. Similar findings with regard to WBC counts were also observed in pigs after per os administration of tulathromycin in a premix form (17). However, in the aforementioned study, only the total WBC was analysed. In contrast, in rabbits treated with tulathromycin, decreased WBC counts were observed. However, only one measurement of rabbit WBC was performed, $6 \mathrm{~h}$ after tulathromycin administration (12). It has also been reported that another compound from the macrolide group called tilmicosin may cause a temporary decrease in the WBC count in rabbits (1). These results suggest that rabbit leukocytes may show species-specific susceptibility to macrolides. Counter to this suggestion, however, is the observation concerning treatment of CD4+ T-cells obtained from healthy humans with azithromycin or clarithromycin macrolides as used in human medicine. This results in inhibition of the CD4+ T-cells proliferation rate and cytokine secretion, which are essential in evoking an effective immune response to pathogens (24).

In contrast, the present findings indicate that beyond its antimicrobial effect, ceftiofur also possesses immunomodulatory properties. The counts of $\mathrm{CD} 3+$, CD21+, CD4+CD8-, CD4-CD8+, and double positive CD4CD8 cell subsets decreased in pigs treated with ceftiofur. It should, however, be noted that the suppressive effect of ceftiofur on blood lymphocyte subpopulations counts was temporary. This may have an unfavourable impact on the immune system response to infection and/or vaccination. This assumption is in accordance with the outcome of earlier studies conducted by Pomorska-Mól et al. $(21,22)$ in which therapeutic doses of ceftiofur during vaccination impaired post-vaccinal immune responses in pigs. The negative effect of ceftiofur, florfenicol, and enrofloxacin on B and T cell subsets was also found in another study on chicks, where results were similar to the present study (6). However, the authors did not analyse the B and T cell subsets in blood, but did so in the lymphatic organs. In addition, the authors also found that this suppressive effect was augmented by E. coli LPS administration and they concluded that all the antimicrobials studied might have an impact on the immune response to bacterial endotoxins in chicks. Earlier studies by the same authors also revealed that ceftiofur used in therapeutic doses affects the number, percentage, and distribution of B cells in the bursa of Fabricius in chicks (5). 

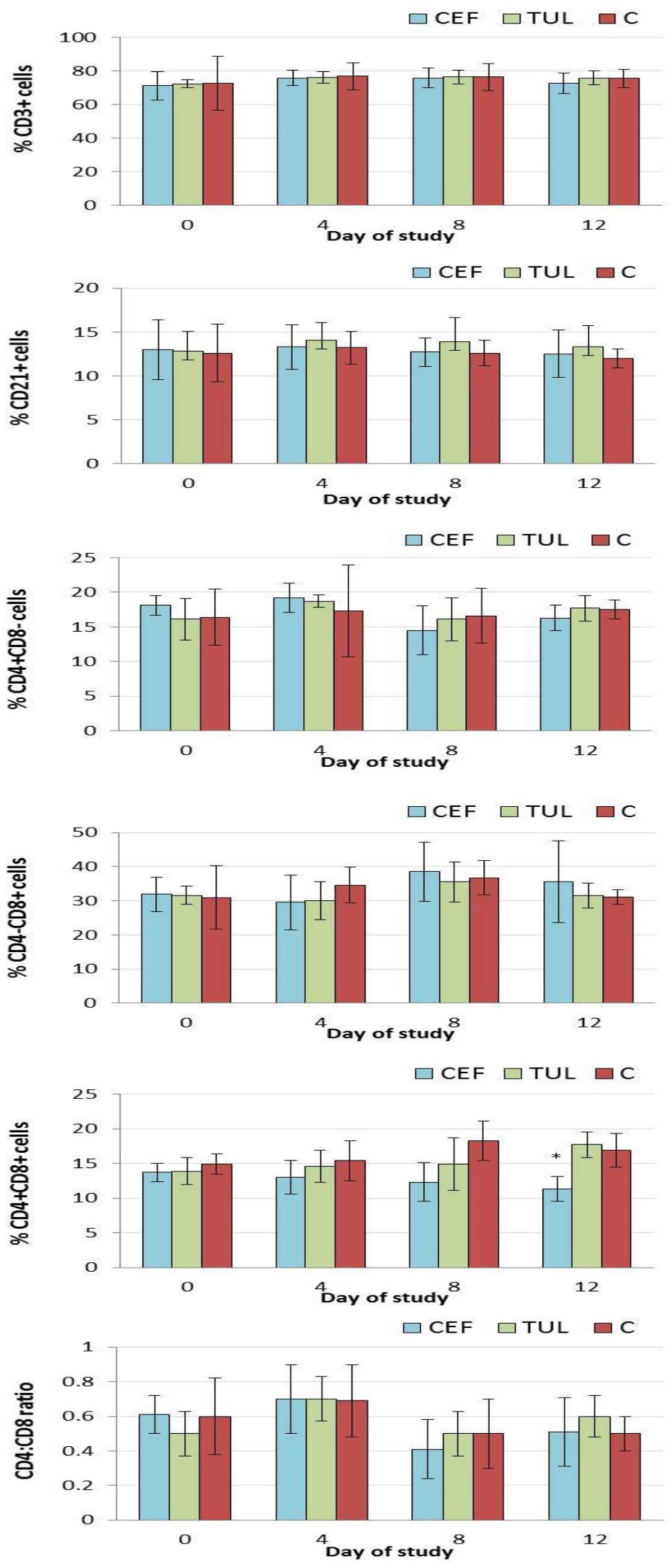

Fig. 1. The mean ( \pm SD) percentage of circulating T-cell subpopulations (CD3+, CD21+, CD4+CD8-, CD4-CD8+, and CD4+CD8+ cells) in pigs from CEF, TUL, and C groups and the CD4:CD8 ratio (calculated as the percentage of CD4+ cells divided by the percentage of CD8+ cells) 

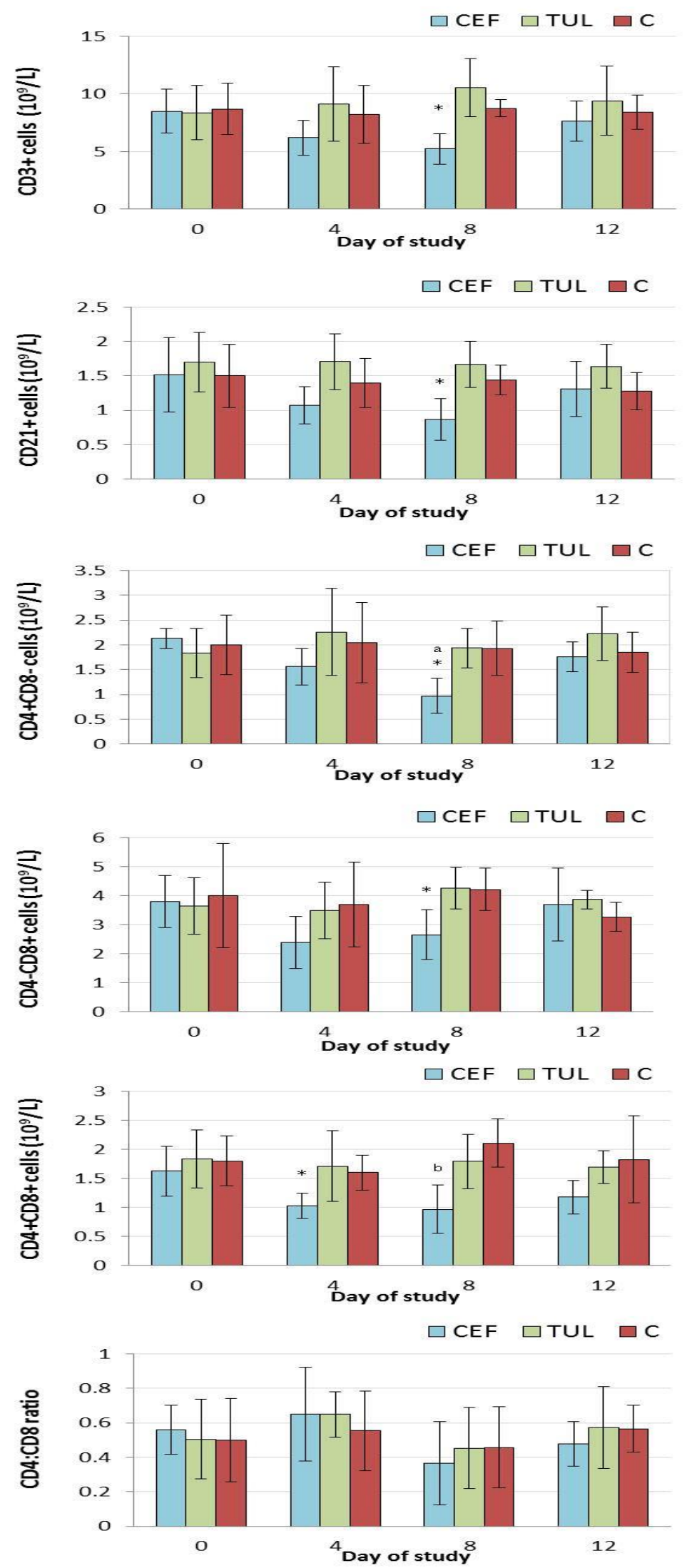

Fig. 2. The mean $( \pm \mathrm{SD})$ number of circulating $\mathrm{T}$-cell subpopulations $(\mathrm{CD} 3+, \mathrm{CD} 21+$, CD4+CD8-, CD4-CD8+, and CD4+CD8+ cells (x 10/L) from CEF, TUL, and C groups and the CD4:CD8 ratio (calculated from the absolute size of the lymphocyte subsets)

* $-\mathrm{P}<0.05$ as compared to $\mathrm{C}$ and TUL group,

a $-\mathrm{P}<0.05$ as compared to day 0 value,

${ }^{b}{ }_{-} \mathrm{P}<0.05$ as compared to $\mathrm{C}$ group 
The authors suggested that this antimicrobial agent may have influence on B cell maturation. It has been documented that this immunosuppressive potential of ceftiofur may be beneficial in resolving bacterial infection. Ceftiofur pretreatment of mice subsequently challenged by LPS significantly decreased plasma levels of tumour necrosis factor- $\alpha$ (TNF- $\alpha$ ), interleukin- $1 \beta$ (IL-1 $\beta$ ), and IL- 6 compared to mice treated with LPS alone (7). According to the authors, downregulation of pro-inflammatory cytokines by ceftiofur decreases mortality in LPS-challenged mice. Similar outcomes were also reported in mice with pulmonary inflammation induced by heat-killed Streptococcus pneumonia and treated with a different cephalosporin (cefodizime) (3). The cytokines are produced by different cell types e.g. monocytes, activated macrophages, NK cells, and T and B cells (2, 23, 25). Thus, based on present results, it may be assumed that through decreasing T-cell subpopulations, ceftiofur may also affect cytokine secretion. However, future studies focusing on the influence of ceftiofur on inflammatory cytokine profile in healthy and challenged pigs and its clinical relevance should be conducted.

In summary, the results of the present study indicate that ceftiofur, irrespective of its antimicrobial properties, transiently suppresses immune response with regard to the absolute number of the $\mathrm{CD} 3+, \mathrm{CD} 21+$, CD4+CD8-, CD4-CD8+, and double positive CD4CD8 cells. The results of this study suggest that the effect of ceftiofur on lymphocyte subpopulations in blood should be taken into consideration before choosing this compound, especially in pigs with impaired immune function (e.g. mycotoxicosis, immunosuppressive viral, and/or bacterial infection and stress conditions).

Conflict of Interests Statement: The authors declare that there is no conflict of interests regarding the publication of this article.

Financial Disclosure Statement: This work was supported by the National Science Centre, Poland within the research grant No. DEC-2012/05/B/NZ7/03114 and by the KNOW (Leading National Research Centre) Scientific Consortium, "Healthy Animal - Safe Food", a division of the Ministry of Science and Higher Education, No. 05-1/KNOW2/2015 (K/02/1.1).

Animal Rights Statement: The authors declare that the experiments on animals were conducted in accordance with local Ethical Committee laws and regulations as regards care and use of laboratory animals. All procedures were approved by the Local Ethical Commission (University of Life Sciences in Lublin, Poland).

\section{References}

1. Altunok V., Yazar E., Elmas M., Traş B., Baş A.L., Cöl, R.: Investigation of haematological and biochemical side effects of tilmicosin in healthy New Zealand rabbits. J Vet Med Series B 2002, 49, 68-70.

2. Bazzoni F., Beutler B.: The tumor necrosis factor ligand and receptor families. New Engl J Med 1996, 334, 1717-1725.

3. Bergeron Y., Ouellet N., Deslauriers A.M., Simard M., Olivier M. Bergeron M.G.: Reduction by cefodizime of the pulmonary inflammatory response induced by heat-killed Streptococcus pneumoniae in mice. Antimicrob Agents Chemother 1998, 42, 2527-2533.

4. Chang C.F., Chang L.C., Chang Y.F., Chen M., Chiang T.S.: Antimicrobial susceptibility of Actinobacillus pleuropneumoniae, Escherichia coli, and Salmonella choleraesuis recovered from Taiwanese swine. J Vet Diagn Invest 2002, 14, 153-157.

5. Chrząstek K., Madej J.P., Mytnik E., Wieliczko A.: The influence of antibiotics on B-cell number, percentage, and distribution in the bursa of Fabricius of newly hatched chicks. Poult Sci 2011, 90, 2723-2729.

6. Chrzastek K., Wieliczko A.: The influence of enrofloxacin, florfenicol, ceftiofur and E. coli LPS interaction on T and B cells subset in chicks. Vet Res Commun 2015, 39, 53-60.

7. Ci X., Li H.Y., Song Y., An Y., Yu Q., Zeng F., Deng X.: Ceftiofur regulates LPS-induced production of cytokines and improves LPS-induced survival rate in mice. Inflammation 2008, 31, 422-427.

8. Clinical and Laboratory Standards Institute. Reference leukocyte (WBC) differential count (proportional) and evaluation of instrument methods; Approved standard 2007, 27.

9. Committee for Veterinary Medicinal Products Summary Report: Ceftiofur. The European Agency for the Evaluation of Medicinal Products EMEA/CVMP/80785/2006-Final.

10. Committee for Veterinary Medicinal Products Summary Report: Tulathromycin. The European Agency for the Evaluation of Medicinal Products EMEA/MRL/842/2002-Final.

11. Duquette S.C., Fischer C.D., Williams A.C., Sajedy S. Feener T.D., Bhargava A., Reti K.L., Muench G.P., Morck D.W., Allison J., Lucas M.J., Buret A.G.: Immunomodulatory effects of tulathromycin on apoptosis, efferocytosis, and proinflammatory leukotriene B4 production in leukocytes from Actinobacillus pleuropneumoniae-or zymosan-challenged pigs. Am J Vet Res 2015, 76, 507-519.

12. Er A., Altan F., Cetin G., Dik B., Elmas M., Yazar E.: Assessment of the cardiotoxicity of tulathromycin in rabbits. Acta Vet Hung 2011, 59, 327-335.

13. Evans N.A.: Tulathromycin: an overview of a new triamilide antibiotic for livestock respiratory disease. Vet Ther 2005, 6, 83-95.

14. Fernandez Filho T., Favaro C Jr., Ingberman M., Beirao B.C.B., Inoue A., Gomes L., Caron L.F.: Effect of spray Escherichia coli vaccine on the immunity of poultry. Avian Dis 2013, 57, 671-676.

15. Fischer C.D., Beatty J.K., Duquette S.C., Morck D.W., Lucas M.J., Buret A.G: Direct and indirect anti-inflammatory effects of tulathromycin in bovine macrophages: inhibition of CXCL-8 secretion, induction of apoptosis, and promotion of efferocytosis. Antimicrob Agents Chemother 2013, 57, 1385-1393.

16. Fischer C.D., Beatty J.K., Zvaigzne C.G., Lucas M.J., Buret A.G.: Anti-inflammatory benefits of antibiotic-induced neutrophil apoptosis: tulathromycin induces caspase-3-dependent neutrophil programmed cell death and inhibits NF-kappaB signaling and CXCL8 transcription. Antimicrob Agents Chemother 2011, 55, 338-348.

17. Hao Z., Zhao Y., Wu H., Hao L., Ding Z., Yang F., Qu B.: The physiological and biochemical evaluation of tulathromycin premix in pigs. J Anim Vet Adv 2012, 11, 4161-4166.

18. Mangano K., Quattrocchi C., Aiello C., Scalia G., Speciale A., Nicoletti G., Di Marco R.: Immunomodulatory properties of cefaclor: in vivo effect on cytokine release and lymphoproliferative response in rats. J Chemother 2006, 18, 641647.

19. Nutsch R.G., Hart F.J., Rooney K.A., Weigel D.J., Kilgore W.R., Skogerboe T.L.: Efficacy of tulathromycin injectable solution for 
the treatment of naturally occurring swine respiratory disease. Vet Ther 2005, 6, 214-224.

20. Pomorska-Mól M., Pejsak Z.: Effect of therapeutic doses of enrofloxacin on circulating lymphocyte subpopulations in pigs. Bull Vet Inst Pulawy 2015, 59, 287-293.

21. Pomorska-Mól M., Czyżewska-Dors E., Kwit K., Wierzchosławki K., Pejsak Z.: Ceftiofur hydrochloride affects the humoral and cellular immune response in pigs after vaccination against swine influenza and pseudorabies. BMC Vet Res 2015, 11, 268.

22. Pomorska-Mól M., Kwit K., Wierzchosławki K., Dors A., Pejsak Z.: The effect of amoxicillin, ceftiofur, doxycycline, tiamulin and tulathromycin on swine humoral immune response induced by erysipelas vaccination. Vet Rec 2016, 178, 559.
23. Pomorska-Mól M., Czyżewska-Dors E., Kwit K., Pejsak Z. Enrofloxacin decreases IL-6 and TNF-á production by lipopolysaccharide-stimulated porcine peripheral blood mononuclear cells. J Vet Res 2016 60, 189-193.

24. Ratzinger F., Haslacher H., Poeppl W., Hoermann G., Kovarik J.J., Jutz S., Steinberger P., Burgmann H., Pickl W.F., Schmetterer KG.: Azithromycin suppresses CD4(+) T-cell activation by direct modulation of mTOR activity. Sci Rep 2014, 4, 7438.

25. Villiger P.M., Cronin M.T., Amenomori T., Wachsman W., Lotz M.: IL-6 production by human T lymphocytes. Expression in HTLV-1-infected but not in normal T cells. J Immunol 1991, 146, 550-559. 